\title{
FORMAS ANÓMALAS EN LOS CORALES EOCENOS DE LA CUENCA DE IGUALADA (NORESTE DE ESPAÑA)
}

\author{
Germán ÁLVAREZ-PÉREZ* y Pere BUSQUETS
}

\author{
Universitat de Barcelona, Facultat de Geologia, Departament d’Estratigrafia, \\ Paleontologia i Geociències Marines. Martí i Franquès s/n. 08028 Barcelona; \\ galvarez@xtec.cat; pere.busquets@ub.edu \\ * Corresponding author
}

\begin{abstract}
Álvarez-Pérez, G. y Busquets, P. 2012. Formas anómalas en los corales eocenos de la Cuenca de Igualada (Noreste de España). [Anomalous forms of the Eocene corals in the Igualada Basin (North-East Spain)]. Revista Española de Paleontología, 27 (1), 15-28. ISSN 0213-6937.
\end{abstract}

\begin{abstract}
The anomalous forms of corals from the Igualada Basin "Upper Eocene", which is located in the Southern Pyrenees and forms part of the Ebro Basin, are described. The corals show anomalies in their shape and in their development and growth. The solitary and colonial corals were analysed separately. In order to account for anomalous forms, the most probable causes were considered with reference to those already accepted by other paleontologists. Our study provides new data for the description and systematic classification of all the coral species in the Igualada basin and other equivalent basins of the Neotethys related to Alpine Orogeny.
\end{abstract}

Keywords: Corals, Igualada basin, Eocene, Bartonian, Priabonian.

\section{RESUMEN}

\begin{abstract}
En este trabajo hemos descrito las formas anómalas que presentan algunos corales de la Cuenca de Igualada (Eoceno Superior), situada al sur de los Pirineos, y que forma parte de la Cuenca del Ebro. Para facilitar dicho estudio hemos agrupado los corales en solitarios y coloniales, analizando por separado las formas anómalas que presentan cada uno de dichos grupos. Al exponer cada una de las formas anómalas hemos hecho referencia a las posibles causas que las hayan provocado y que muchas veces son difíciles de determinar. En este trabajo nos hemos centrado sobre todo en aquellas que consideramos como más probables y que han sido aceptadas por otros paleontólogos estudiosos del tema. Este trabajo aporta nuevos datos para la descripción y clasificación sistemática de las especies coralinas, no sólo de la zona de Igualada sino también del resto de cuencas equivalentes del Neotethys relacionadas con el orógeno alpino.
\end{abstract}

Palabras clave: Corales, Cuenca de Igualada, Eoceno, Bartoniense, Priaboniense.

\section{INTRODUCCIÓN}

Recientemente, en la clasificación de los corales, se han incorporado nuevos conceptos, siendo uno de ellos las diversas formas que los individuos de una misma especie adquieren a lo largo de su crecimiento y desarrollo y que han quedado reflejadas en su esqueleto (Best et al., 2003; Zlatarski, 2007, 2010).

Los individuos de una misma especie tienden a adquirir una determinada forma. Cualquier otra forma que difiera de ella será una anomalía. Estas anomalías pueden observarse a nivel de individuo, a nivel de población o a nivel de especie (Zlatarski \& Martínez-Estalella, 1982).
Dichas anomalías muestran no sólo la variabilidad de estos organismos sino también su habilidad para reaccionar ante las condiciones adversas y adaptarse a las mismas para lograr sobrevivir (Zlatarski, 2010).

Las causas que provocan estas anomalías son muchas. Unas se pueden considerar internas, ya que dependen fundamentalmente del propio individuo o colonia, y otras se pueden considerar externas, ya que dependen de la interacción con los elementos del medio en el que vive el coral. Como causas internas consideramos las mutaciones y las enfermedades contraídas por el coral. Como causas externas consideramos las condiciones físico-químicas del agua y la interacción con los seres vivos que habitan en el entorno del coral (Zlatarski \& Martínez-Estalella, 1982; Rosen et al., 2002). 
En la Tabla 1 presentamos la lista de los agentes y las causas que provocan estas anomalías.

Tabla 1. Causas de las anomalías y agentes que las producen. Causes of the anomalies and agents that produce.

\begin{tabular}{|c|c|c|c|}
\hline \multicolumn{2}{|c|}{ CAUSAS } & \multicolumn{2}{|c|}{ AGENTES } \\
\hline \multirow{2}{*}{ Internas } & Mutaciones & & \\
\hline & Enfermedades & & \\
\hline \multirow{15}{*}{ Externas } & \multirow{7}{*}{ Físicas } & \multirow{5}{*}{ Agua } & Temperatura \\
\hline & & & Turbidez \\
\hline & & & Profundidad \\
\hline & & & Oleaje \\
\hline & & & Corrientes \\
\hline & & \multicolumn{2}{|c|}{ Sedimentos } \\
\hline & & \multicolumn{2}{|c|}{ Espacio disponible } \\
\hline & \multirow{3}{*}{ Químicas } & \multirow{3}{*}{ Agua } & Salinidad \\
\hline & & & Acidez \\
\hline & & & Contaminación \\
\hline & \multirow{5}{*}{ Seres vivos } & Beneficiosos & Simbiontes \\
\hline & & \multirow{3}{*}{ Nocivos } & Parásitos \\
\hline & & & Depredadores \\
\hline & & & Competidores \\
\hline & & Indiferentes & Comensales \\
\hline
\end{tabular}

No siempre es fácil determinar la causa que ha provocado una anomalía y mucho menos determinar el agente que la ha producido, ya que, en la mayoría de los casos, no se dispone de suficientes datos para ello (Best et al., 2003). Además, no debe olvidarse que una determinada causa puede ser producida por distintos agentes (Zlataraski, 2010).

\section{ESTRATIGRAFÍA DE LA CUENCA DE IGUALADA}

La Cuenca de Igualada forma parte de la Cuenca del Ebro, que constituye una cuenca de antepaís relacionada con el orógeno de los Pirineos (Fig. 1).

A lo largo del Paleoceno y del Eoceno tuvieron lugar cuatro eventos que configuraron cuatro unidades tectoestratigráficas (Tabla 2).

El primer evento tuvo lugar durante el Ilerdiense y el Cuisiense Inferior. Se extiende desde la SBZ (Shallow Bentonic Zone) 5 a la SBZ 10. Este evento está representado por las calizas con miliólidos de la Formación Orpí y por los materiales detríticos del intervalo inferior de la Formación Pontils. En la base de esta primera unidad tectoestrati-

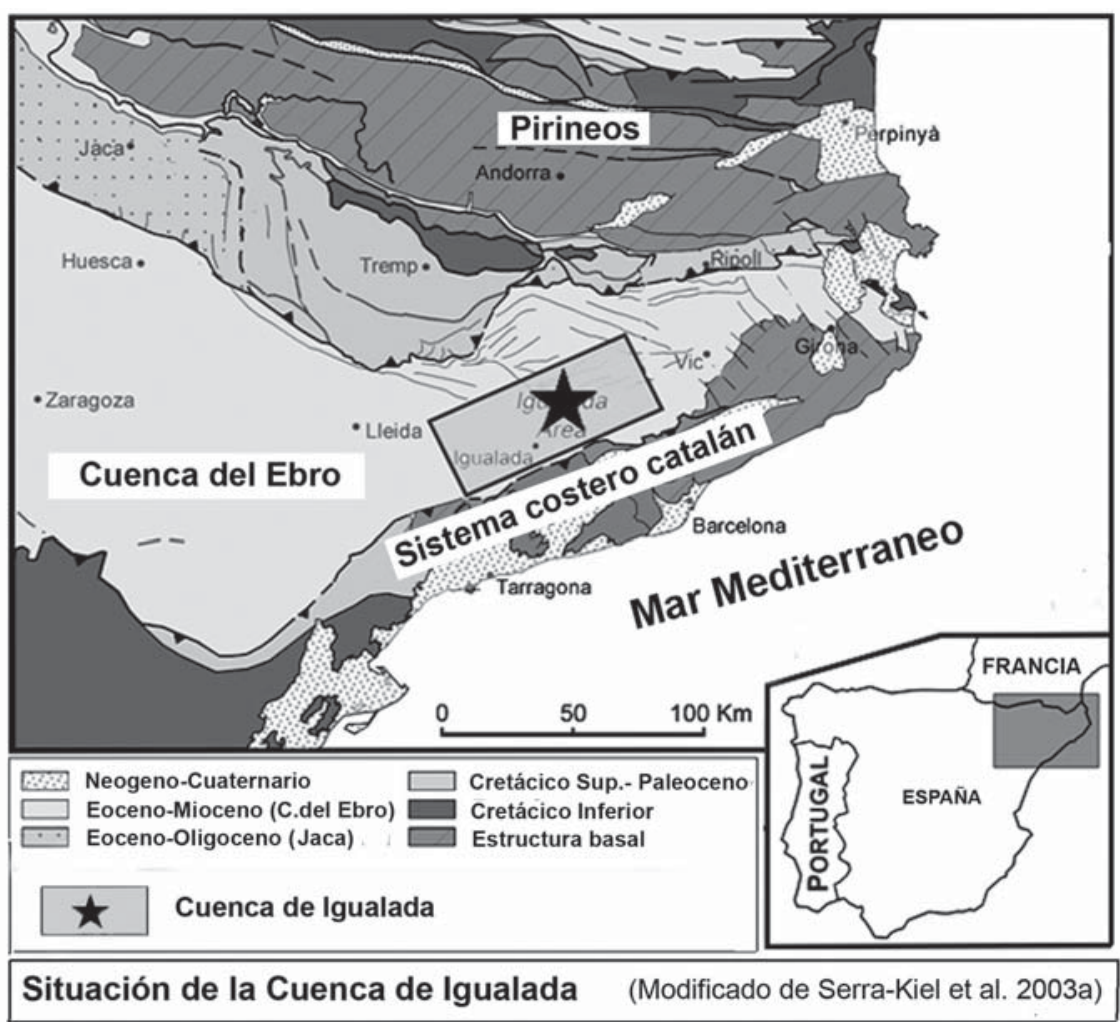

Figura 1. Situación geológica de la Cuenca de Igualada, modificado de Serra-Kiel et al. (2003).

Geological location of the Igualada Basin, modified from Serra-Kiel et al. (2003). 
Tabla 2. Estratigrafía de la Cuenca de Igualada, según datos de Serra-Kiel et al.(2003a) y de Costa et al. (2009). Stratigraphy of the Igualada Basin according to Serra-Kiel et al. (2003a) and to Costa et al. (2009).

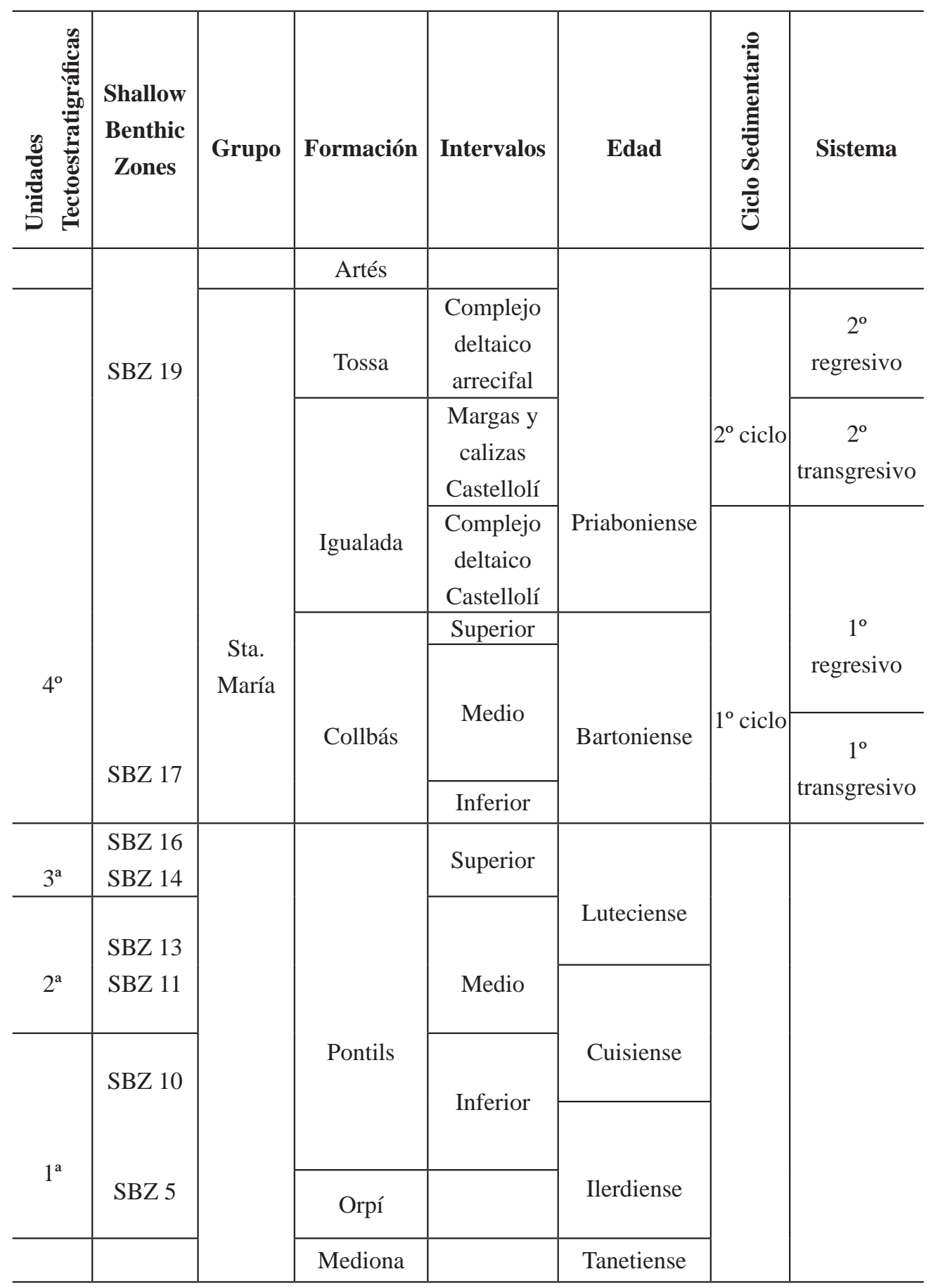

gráfica se encuentran las calizas (mudstone) pertenecientes a la Formación Mediona (Tanetiense Superior).

El segundo evento tuvo lugar durante el Cuisiense Medio y Superior y el Luteciense Inferior. Comprende desde la SBZ 11 a la SBZ 13. Está representado por los sedimentos detríticos del intervalo medio de la Formación Pontils.

El tercer evento tuvo lugar durante el Luteciense Medio y Superior. Comprende desde la SBZ 14 a la SBZ 16.
Está representado por los sedimentos detríticos del intervalo superior de la Formación Pontils.

El cuarto evento tuvo lugar durante el Bartoniense y el Priaboniense. Comprende desde la SBZ 17 a la SBZ 19. Está representado por el Grupo de Sta. María que comprende las formaciones de Collbás, de Igualada y de La Tossa (Fig. 2) (Alvarez Pérez et al., 1995; Serra-Kiel et al., 2003a, b; Cascella \& Dinarès-Turell, 2009; Costa et al., 2009). 


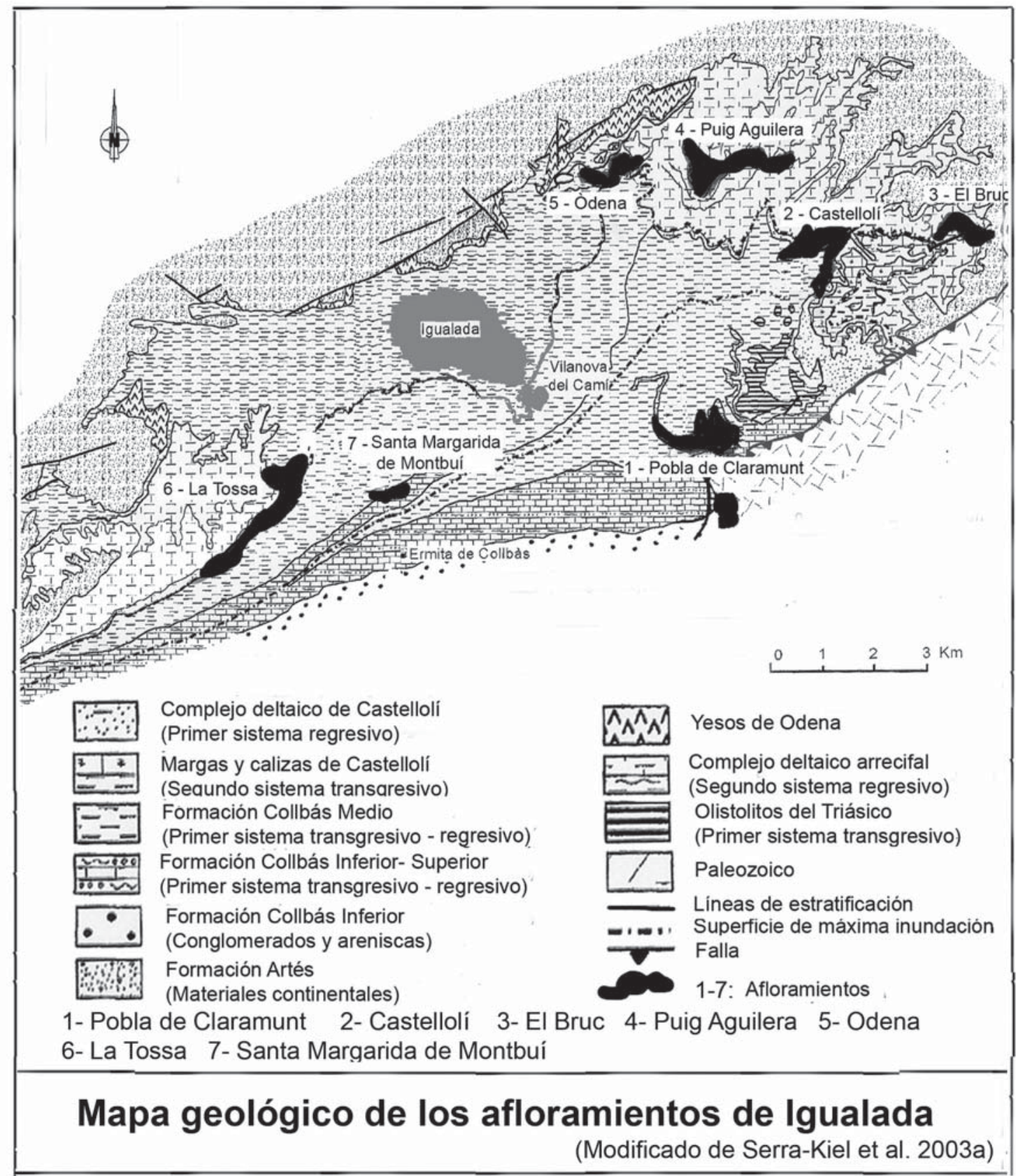

Figura 2. Mapa geológico y afloramientos estudiados del área de Igualada, modificado de Serra-Kiel et al. (2003). Geological map and studied outcrops of the Igualada area, modified from Serra-Kiel et al. (2003).

\section{GRUPO DE SANTA MARÍA}

El Grupo de Santa María se caracteriza por la existencia de dos grandes ciclos sedimentarios transgresivosregresivos.

El primer ciclo sedimentario se inicia con una transgresión de materiales marinos depositados durante el intervalo inferior y el inicio del intervalo medio de la Formación Collbás, y termina con una regresión de materiales marinos y continentales depositados en los intervalos medio y superior de la Formació Collbás y en el intervalo del Complejo deltaico de Castellolí.
El segundo ciclo sedimentario empieza con una transgresión en la que los sedimentos marinos, representados por las margas y calizas de Castellolí, se situan encima de los materiales continentales del Complejo deltaico de Castellolí. La regresión correspondiente a este ciclo está representada por los materiales del complejo deltaicoarrecifal de la Formación Tossa. En dicha regresión aparecen diversos episodios transgresivos donde los sedimentos continentales son colonizados por bioconstrucciones algo-coralinas.

Al final de dichos ciclos sedimentarios aparecen los materiales siliciclásticos de la Formación Artés (Tabla 2). 
En los sistemas transgresivos de dichos ciclos se encuentran formaciones algo-coralinas así como algunos corales solitarios y en los sistemas regresivos aparecen bioconstrucciones algo-coralinas. Las facies coralinas más abundantes y con mayor diversidad de especies se encuentran intercaladas entre los sedimentos deltaicos del segundo sistema regresivo de la Formación Tossa (LópezBlanco, 2006).

\section{MATERIAL ESTUDIADO}

Los ejemplares estudiados han sido recolectados en siete afloramientos distintos (Fig. 2). Los afloramientos de La Pobla de Claramunt (1) y de Santa Margarita de Montbuí (7) pertenecen a la Formación de Collbàs (Bartoniense). Los afloramientos de Castellolí (2), de El Bruc (3) y de Odena (5) pertenecen a la Formación de Igualada (Priaboniense). Los afloramientos de Puig Aguilera (4) y de La Tossa (6) pertenecen a la Formación Tossa (Priaboniense).

La mayoría de ejemplares aparecen aislados de modo que han conservado su individualidad facilitando así su análisis y estudio. Todos los ejemplares han sufrido una diagénesis que ha transformado el aragonito original de su esqueleto en calcita. Con ello, han desaparecido las microestructuras y únicamente se han conservado las formas externas, que son precisamente las que reflejan las anomalías aquí estudiadas. En el texto de cada figura se indica la especie a que pertenece el ejemplar figurado, el afloramiento donde ha sido encontrado y su catalogación dentro de la colección de Germán Alvarez (GA).

\section{MÉTODO DE TRABAJO}

Para facilitar el estudio de las anomalías hemos reunido los corales en dos grandes grupos: solitarios y coloniales. Dentro del primero grupo hemos separado los ejemplares trocoides de los discoidales.

Así mismo, hemos considerado por separado las anomalías propias de la forma del coral de aquellas que se refieren a su crecimiento y desarrollo.

\section{ANOMALÍAS EN LOS CORALES SOLITARIOS}

\section{ANOMALÍAS EN LA FORMA}

En todo coral solitario hay que tener en cuenta la forma del cáliz y la forma del propio individuo.

Corales trocoides

Anomalías en la forma del cáliz
Los corales trocoides desarrollan normalmente un cáliz de forma circular ya que su tendencia al crecimiento es igual en todas las direcciones (Fig. 3a). Por tanto, todo cáliz que no sea circular será considerado como anómalo.

Observando diversos ejemplares de Placosmiliopsis bilobatus (d'Achiardi, 1867), hemos encontrado individuos con el cáliz elíptico (Fig. 3b); individuos cuyo cáliz tiene dos invaginaciones laterales que le confieren la forma de un "ocho" (Fig. 3c); e individuos cuyo cáliz posee varias invaginaciones que le dan una forma irregular (Fig. 3d).

Anomalías en la forma del individuo

Los corales trocoides tienen una forma cónica regular invertida (Figs 3f, g).

Analizando diversos ejemplares de Placosmiliopsis bilobatus, encontramos individuos rectos (Figs 3f, g) e individuos curvados (Fig. 3h). También encontramos individuos que son bajos (Fig. 3e) y otros que son altos (Figs 3f, g, h). Finalmente los individuos pueden ser anchos (Fig. 3e) o estrechos (Figs 3g, h). La anchura del coral depende del valor del ángulo de la base. Si es mayor de $90^{\circ}$ diremos que el coral es ancho (Fig. 3e); si es próximo a los $90^{\circ}$, diremos que el coral es medianamente ancho (Fig. 3f) y si es inferior a los $90^{\circ}$, diremos que el coral es estrecho (Figs 3g, h).

\section{Corales discoidales}

Los corales discoidales tienen el cáliz de forma circular (Fig. 3j). La cara inferior es plana y la cara superior es ligeramente convexa (Figs 3n, o).

Anomalías en la forma del cáliz

Observando diversos ejemplares de Funginellastraea barcelonensis (Oppenheim, 1911) encontramos individuos que tiene el cáliz ligeramente elíptico (Fig. 3k) y otros con algunas invaginaciones laterales que le confieren un aspecto ondulado (Fig. 3m).

Anomalías en la forma del individuo

Los corales discoidales se diferencian por su grosor, sin que ninguna forma en concreto se pueda considerar como normal y las restantes como anómalas. En ejemplares de Funginellastraea barcelonensis encontramos individuos finos (Fig. 3n) e individuos gruesos (Fig. 3o).

En ejemplares de Nicaeotrochus cyclolitoides (Michelin, 1846) encontramos individuos más gruesos de lo normal, que tienen forma cilíndrica (Fig. 3p).

Estas formas se pueden encontrar en individuos de otras especies similares. Su presencia y la frecuencia con que aparece cada una de ellas dependen de las condiciones ambientales en que vive el coral.

El hecho de encontrar formas intermedias nos hace suponer que el origen de dichas anomalías radica en el genotipo de cada individuo y, por tanto, que forman parte de la variabilidad intraespecífica (Best et al., 2003).

Según Pfister (1985) la presencia de estas formas indica unas condiciones ambientales más o menos tranquilas y estables. 

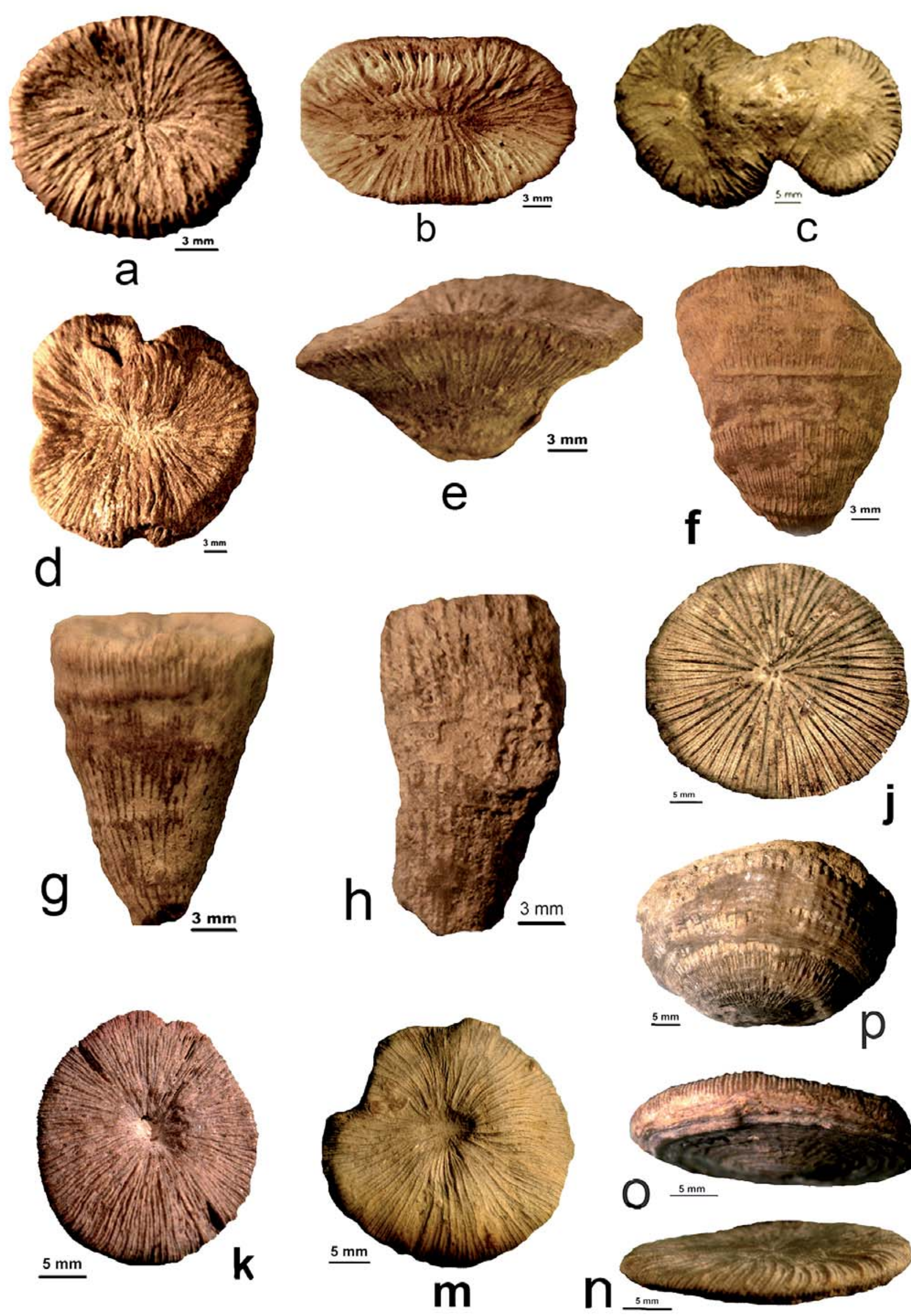


\section{ANOMALÍAS EN EL CRECIMIENTO Y DESARROLLO}

\section{Corales trocoides}

Grosor del individuo

Los corales trocoides, a medida que crecen, se ensanchan de manera regular adquiriendo la forma de un cono invertido (Figs 3f, g).

Cuando la cantidad de sedimento que llega a la zona aumenta de manera significativa, el coral se estrecha y alarga a fin de crecer rápidamente hacia arriba y no quedar enterrado por el sedimento. Este tipo de estrechamiento puede observarse en ejemplares de Plascomiliopsis bilobatus (Figs 4a, b).

Por el contrario, si el aporte detrítico disminuye, el individuo se ensancha para aprovechar mejor las condiciones favorables del exterior, tal como puede verse en un ejemplar de Pattalophyllia grumi (Catullo, 1852) en el que observamos un ligero ensanchamiento en la parte superior del mismo (Fig. 4c) (Gameil, 2005).

Cuando estas variaciones en el aporte de sedimento alternan entre sí, el individuo presenta sucesivos estrechamientos y ensanchamientos, como respuesta a dichas fluctuaciones, tal como podemos observar en un ejemplar de Pattalophyllia grumi (Fig. 4d) (Best et al., 2003; Gameil, 2005).

\section{Necrosis}

El coral puede sufrir una necrosis parcial del cáliz. En el mejor de los casos, seguirá desarrollándose a partir de la parte viva que queda, pero en su esqueleto quedará constancia de la mutilación recibida, como puede verse en el ejemplar de Plascomiliopsis bilobatus de la figura 4e. Las posibles causas de dicha necrosis son: una enfermedad, un parásito, la depredación, o el enterramiento parcial del coral por el sedimento (Zlatarski, 2010).

\section{Geniculación}

Los corales trocoides pueden presentar una curvatura muy pronunciada denominada geniculación. Es el caso del ejemplar de Plascomiliopsis bilobatus de la figura 4f.
La geniculación es un proceso que empieza cuando un individuo que vive anclado en un fondo blando cae y queda tumbado. Si el individuo no queda enterrado por el sedimento, continua creciendo en sentido vertical. Este es el caso del ejemplar de Leptomussa variabilis d'Achiardi, 1867 que inicialmente vivía en posición vertical (Fig. 4g), cayó sobre el fondo y quedó tumbado (Fig. 4h) pero siguió creciendo hacia arriba y dio lugar a la geniculación formada por la parte antigua del coral y la de nueva formación (Fig. 4j) (Alvarez Pérez, 1993).

La causa de la caída del coral puede ser el roce con un animal bentónico, el oleaje producido por una tempestad o una simple corriente marina (Gameil, 2005).

\section{Corales discoidales}

Anomalías en la cara superior

Si el aporte detrítico es abundante el individuo desarrolla más la zona central de la cara superior que los bordes de la misma, formando así un pequeño promontorio central de pendiente más pronunciada que facilita la expulsión del sedimento que le cae encima. La cara adquiere, por tanto, una forma cóncava. Este es el caso del ejemplar de Funginellastraea barcelonensis de la figura 4k (Gameil, 2005).

Anomalías en la cara inferior

Si el sedimento es muy fino, el individuo desarrolla en la cara inferior una epiteca en forma de anillos concéntricos que impiden que el sedimento penetre en su interior y acabe provocando su muerte. Un ejemplo de ello lo constituye el ejemplar de Funginellastraea barcelonensis de la figura 4m (Gameil, 2005).

\section{Necrosis}

El coral puede sufrir una necrosis parcial del tejido vivo. En ocasiones, debido al ataque de un depredador, sufre también la destrucción del esqueleto de la zona afectada, como el ejemplar de Funginellastraea barcelonensis de la figura 4n, el cual, a pesar de ello, siguió desarrollándose a partir de la parte viva que le quedaba, llegando incluso a regenerar el individuo entero (Fig. 4n) (Alvarez Pérez, 1993).

Figura 3. Anomalías en la forma de los corales solitarios. Corales trocoides. Placosmiliopsis bilobatus. a: (GA 11072b) Puig Aguilera; cáliz circular; b: (GA 6952) Puig Aguilera; cáliz elíptico; c: (GA 7054) Puig Aguilera; cáliz con invaginaciones laterales; d: (GA 11593) Puig Aguilera; cáliz irregular; e: (GA 6968) Puig Aguilera; ejemplar recto, bajo y ancho; f: (GA 6876) Castellolí; ejemplar recto, alto y ancho; g: (GA 12999b) Puig Aguilera; ejemplar recto, alto y estrecho; h: (GA 13093) Castellolí; ejemplar curvado, alto y estrecho. Corales discoidales. Funginellastraea barcelonensis. j: (GA 5495) Puig Aguilera; cáliz circular; k: (GA 5394) El Bruc; cáliz elíptico; m: (GA 5472) Puig Aguilera; cáliz ondulado; n: (GA 5199) Castellolí; ejemplar delgado; o: (GA 5384) Puig Aguilera; ejemplar grueso. Nicaeotrochus cyclolitoides; p: (GA 4999) Puig Aguilera; ejemplar cilíndrico.

Anomaly in the forms of solitary Coral. Trochoids corals. Placosmiliopsis bilobatus. a: (GA 11072b) Puig Aguilera; circular calyx; b: (GA 6952) Puig Aguilera; elliptic calyx; c: (GA 7054) Puig Aguilera; calyx with lateral invaginations; d: (GA 11593) Puig Aguilera; irregular calyx; e: (GA 6968) Puig Aguilera; straight, short and wide specimen; f: (GA 6876) Castellolí; straight, high and wide specimen; g: (GA 12999b) Puig Aguilera; straight, high and narrow specimen; h: (GA 13093) Castellolí; curved, high and narrow specimen. Discoids corals. Funginellastraea barcelonensis. j: (GA 5495) Puig Aguilera; circular calyx; k: (GA 5394) El Bruc; elliptic calyx; m: (GA 5472) Puig Aguilera; undulating calyx; n: (GA 5199) Castellolí; thin specimen; o: (GA 5384) Puig Aguilera; thick specimen. Nicaeotrochus cyclolitoides. p: (GA 4999) Puig Aguilera; cylindrical specimen. 

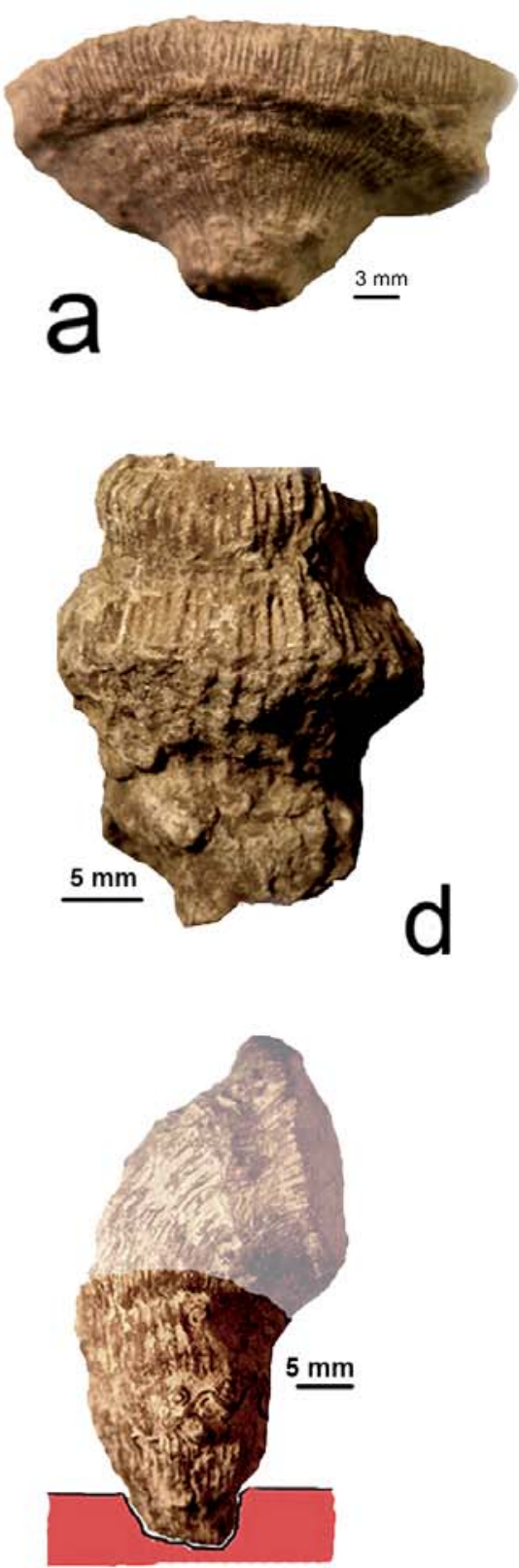

g

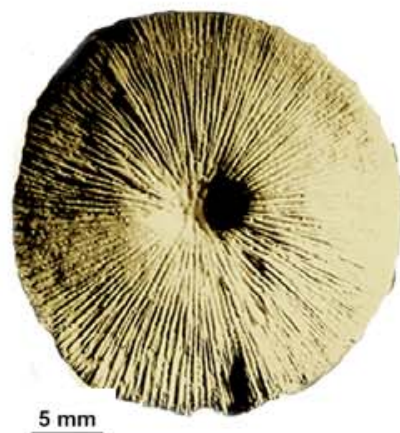

k
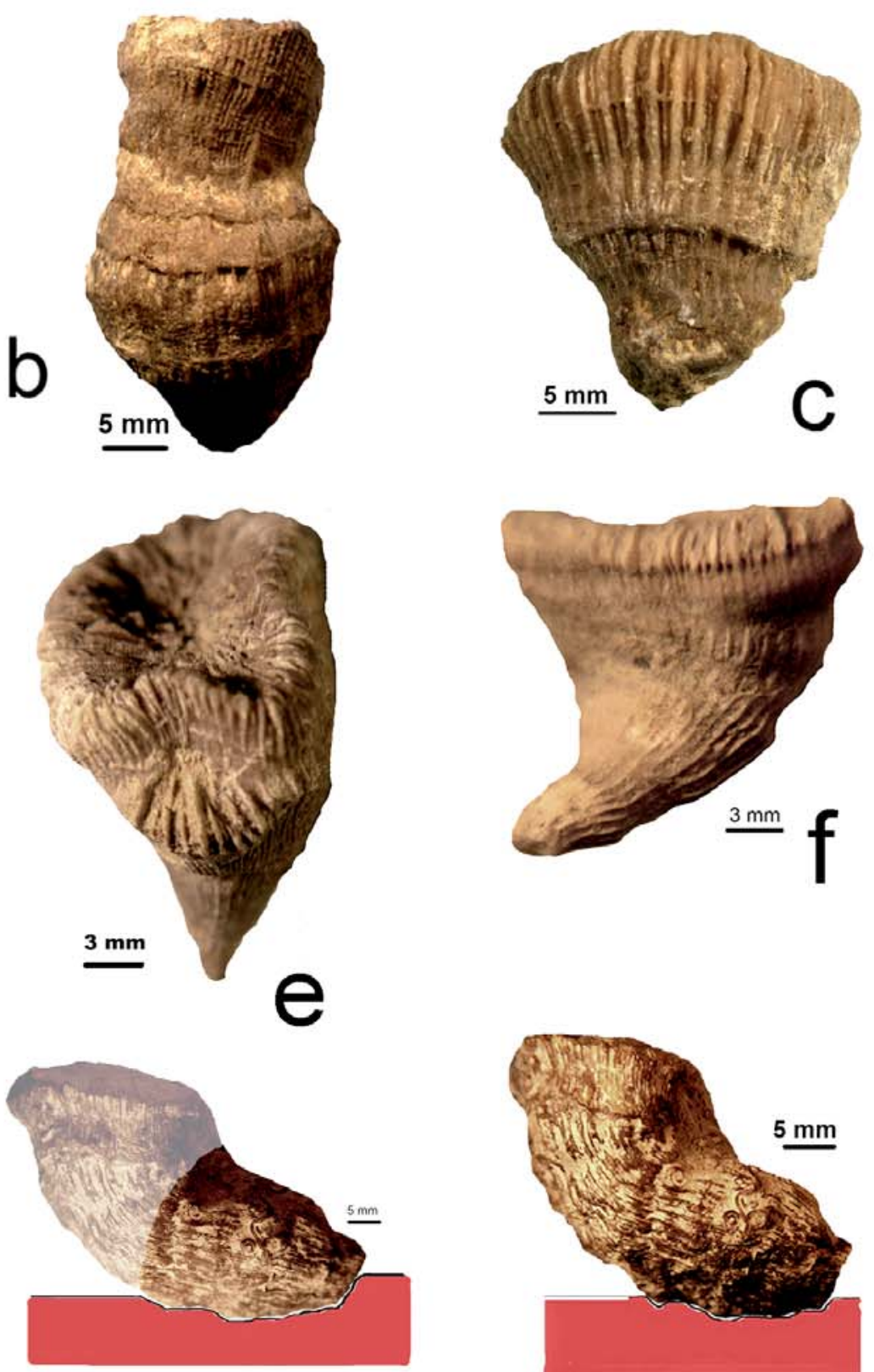

h

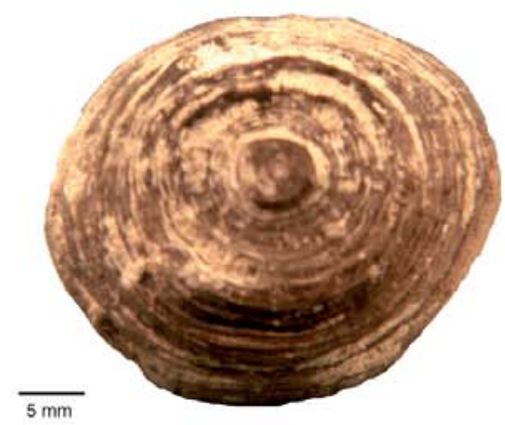

$\mathrm{m}$

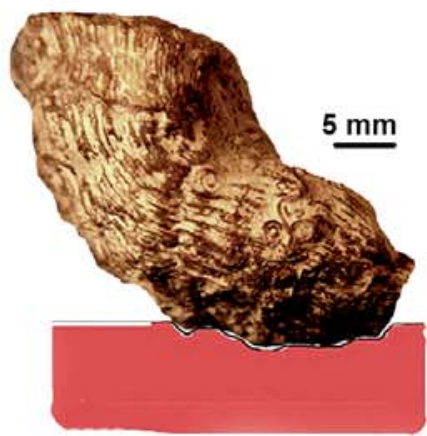

j
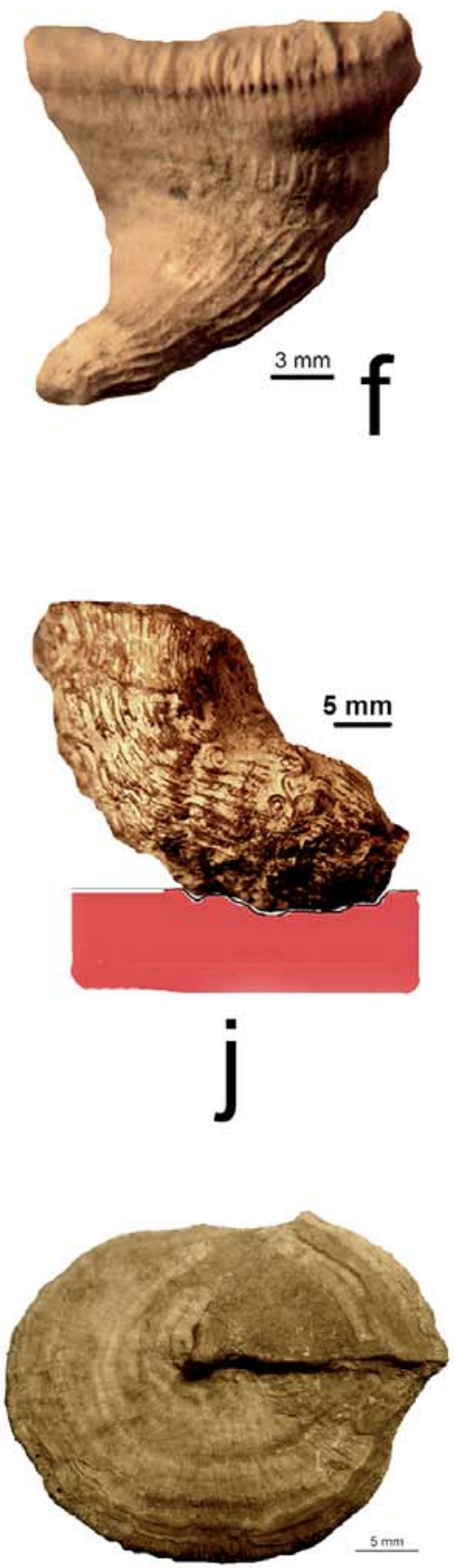

$n$ 


\section{ANOMALÍAS EN LOS CORALES COLONIALES}

Las especies coloniales están muy condicionadas por el medio ambiente en el que viven, reflejando fácilmente los cambios que se producen en dicho medio. El esqueleto de dichas especies refleja claramente si las condiciones ambientales le eran favorables o no.

\section{ANOMALÍAS EN LA FORMA}

\section{Anomalías intracoloniales}

Se refieren fundamentalmente a la forma y a la distribución de las coralitas (Rodríguez \& Gómez-Herguedas, 2002).

\section{Forma de las coralitas}

Una colonia puede tener las coralitas aproximadamente iguales en cuanto a su forma y tamaño, como la colonia de Astrocoenia lobatorotundata (Michelin, 1842) de la figura 5 a.

Las coralitas pueden ser iguales en la forma pero desiguales en el tamaño, como ocurre en la colonia de Astreopora tecta (Catullo, 1856) de la figura 5b. Este caso se da en las especies que tienen gemación intercalicinal. Las coralitas grandes corresponden a individuos adultos ya desarrollados, y las coralitas pequeñas corresponden a individuos jóvenes en vías de desarrollo.

Finalmente, las coralitas pueden ser distintas en la forma y en el tamaño sin seguir una pauta determinada, como el ejemplar de Antiguastraea cvijici (Oppenheim, 1906) de la figura 5c (Zlatarski \& Martínez-Estalella, 1982).

Coralitas "gigantes"

Zlatarski \& Peters (2007) explican cómo algunos ejemplares presentan algunas coralitas de tamaño mucho mayor que las restantes coralitas de la colonia, llamándolas, por ello, "Giant corallite (GC)”. Dichas coralitas presentan un mayor número de septos, como el ejemplar de Astrocoenia lobatorotundata de la figura $5 \mathrm{~d}$.
Esta anomalía ha sido detectada en diversas especies, si bien se desconoce el origen de estas coralitas gigantes. También se desconoce si dichas coralitas tienen una función específica dentro de la colonia.

Distribución de las coralitas

Las coralitas de una colonia pueden estar distribuidas regularmente en toda la superficie de la colonia (Fig. 5e) o pueden estar distribuidas de forma irregular (Fig. 5f).

Anomalías intrapoblacionales e intraespecíficas

Tamaño de las coralitas

Comparando distintas colonias de una misma especie, se pueden encontrar colonias con las coralitas relativamente pequeñas (Fig. $5 g$ ) y colonias con las coralitas de tamaño mucho mayor (Fig. 5h). Esta diferencia se debe a que las condiciones ambientales son más favorables en el segundo caso que en el primero.

Resalte de las coralitas

En las colonias meandroides las series de coralitas pueden estar más o menos resaltadas. Así, en el ejemplar de Cyathoseris castroi (Mallada, 1887) de la figura 5j, las series de coralitas están poco resaltadas mientras que en el ejemplar de la figura 5k lo están mucho más.

\section{ANOMALÍAS EN EL CRECIMIENTO Y DESARROLLO}

\section{Anomalías intracoloniales}

\section{Rotación pasiva}

Algunas colonias, que viven libres en substratos blandos, están sometidas a determinados agentes físicos que las hacen rodar continuamente. En estos casos las coralitas crecen radialmente en todas direcciones, de manera que la colonia adquiere una forma esférica o ligeramente ovalada como el ejemplar de la figura 6a, que corresponde a la especie Stylocoenia macrostyla Reuss, 1870. Dichas colonias carecen de base y de techo (Sorauf, 2010).

Se admite que las causas que provocan la rotación pasiva son las corrientes producidas por las olas o por las mareas, descartando la posible acción de seres vivos (Fabricius, 1964; Sorauf, 2010).

Figura 4. Anomalías en el crecimiento y desarrollo de los corales solitarios Corales trocoides. Placosmiliopsis bilobatus. a: (GA 6497) Castellolí; estrechamiento vertical. b: (GA 13329) Castellolí; estrechamiento vertical. Pattalophyllia grumi. c: (GA 8267) Puig Aguilera; ensanchamiento vertical; d: (GA 8226b) El Bruc; sucesivos entrechamientos y ensanchamientos. Placosmiliopsis bilobatus. e: (GA 11135a) Pobla de Claramunt; regeneración del cáliz; f: (GA 7049) Puig Aguilera; geniculación. Leptomussa variabilis. g: (GA 6229) Puig Aguilera; crecimiento vertical; h: coral tumbado; j: geniculación. Corales discoidales. Funginellastraea barcelonensis. k: (GA 5381) Puig Aguilera; superfície calicinal cóncava. Nicaeotrochus cyclolitoides. m: (GA 5175) Puig Aguilera; epiteca de anillos concéntricos. Funginellastraea barcelonensis. n: (GA 5502) Puig Aguilera; regeneración del individuo.

Anomaly in the growth and development of solitary corals. Trochoids corals. Placosmiliopsis bilobatus. a: (GA 6497) Castellolí; vertical narrowing; b: (GA 13329) Castellolí; vertical narrowing. Pattalophyllia grumi. c: (GA 8267) Puig Aguilera; vertical widening; $\boldsymbol{d}$ : (GA 8226b) El Bruc; successive narrowings and widenings. Placosmiliopsis bilobatus. e: (GA 11135a) Pobla de Claramunt; regeneration of the calice; $f$ : (GA 7049) Puig Aguilera; geniculation. Leptomussa variabilis. g: (GA 6229) Puig Aguilera; vertical growth; h: fallen coral; $\mathbf{j}$ : geniculation. Discoids corals. Funginellastraea barcelonensis. k: (GA 5381) Puig Aguilera; calicular platform concave. Nicaeotrochus cyclolitoides. m: (GA 5175) Puig Aguilera; epitheca in annular rings. Funginellastraea barcelonensis. n: (GA 5502) Puig Aguilera; regeneration of the polyp. 

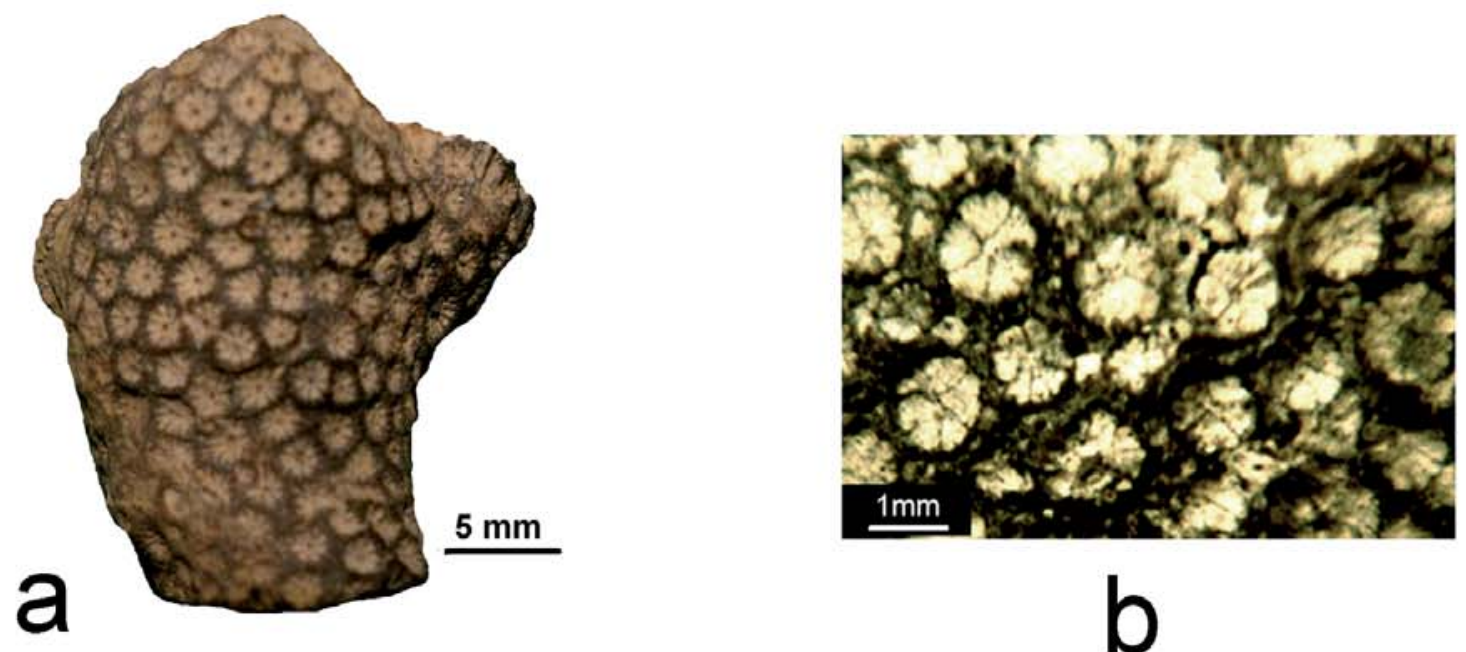

b
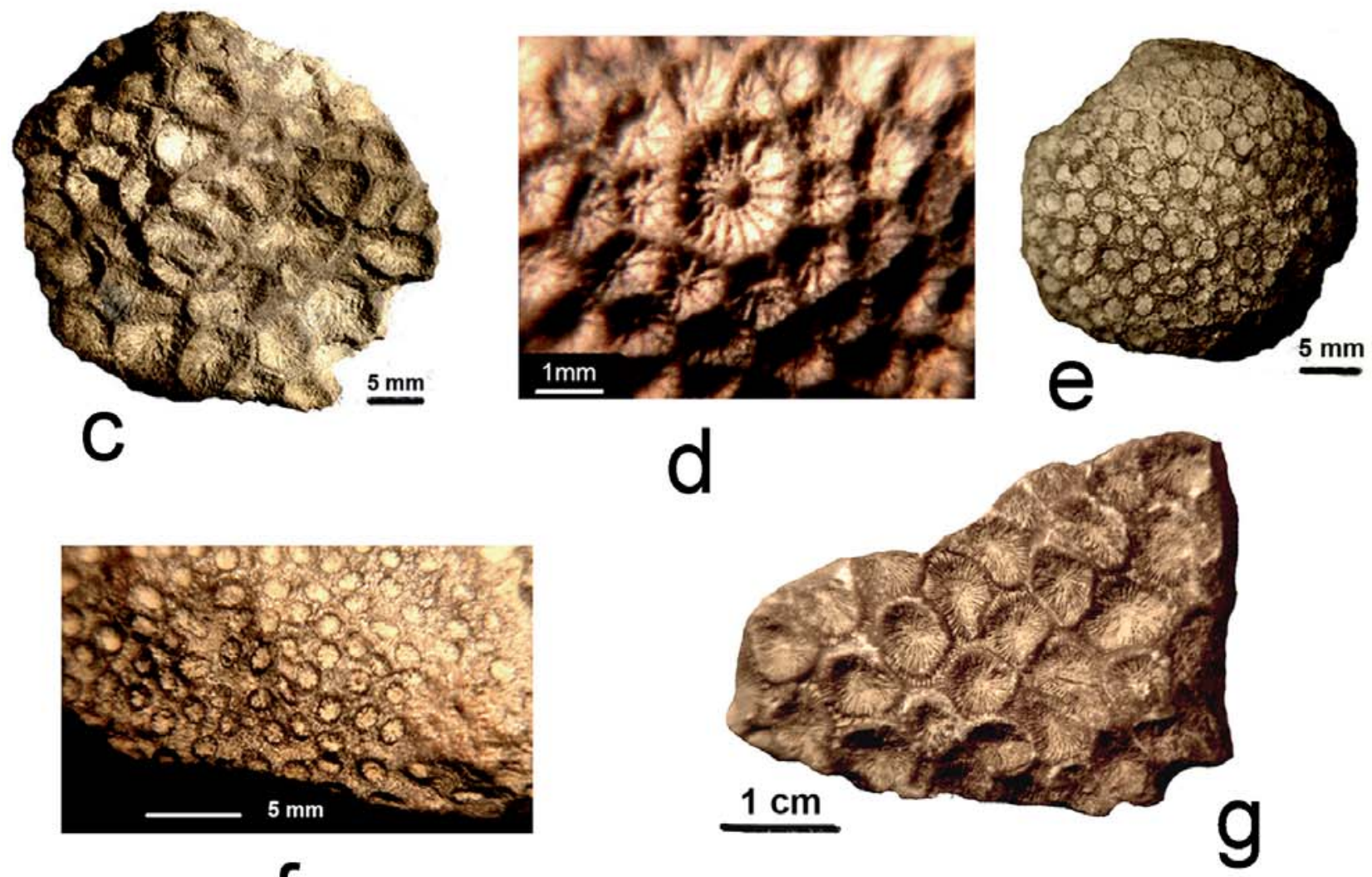

f
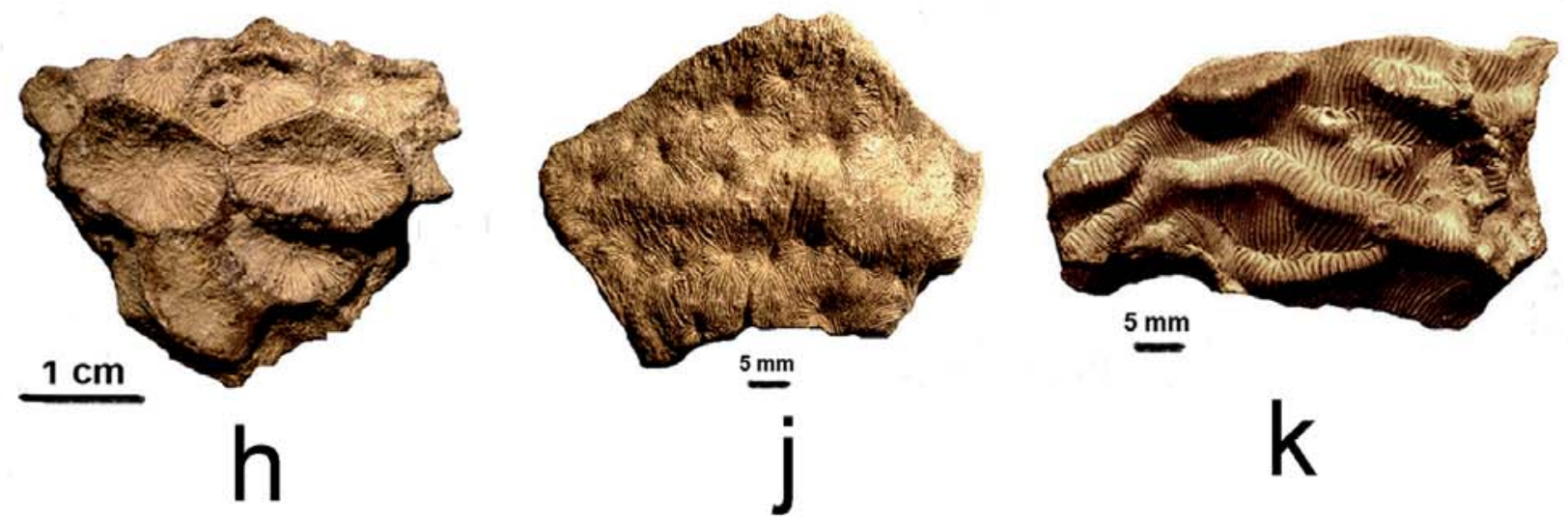
Falta de espacio

Una colonia fijada a un substrato duro, puede encontrar un obstáculo que le impida crecer en una determinada dirección. En tal caso se desarrolla y extiende por la zona libre de obstáculos adquiriendo así una forma desigual. Este es el caso de la colonia de Colpophyllia stellata (Catullo, 1856) de las figuras 6b y c.

Si el obstáculo no la deja crecer en sentido horizontal, lo hará en sentido vertical como el ejemplar de Ellipsocoenia bauzai (Mallada, 1887) de la figura 6d (Pfister, 1985).

\section{Tumores}

Los corales coloniales sufren lesiones provocadas por la depredación de algunos peces que con sus potentes mandíbulas y sus afilados dientes no solamente lesionan el tejido vivo del coral sino que también roen el esqueleto subyacente (Jayewardene et al., 2009).

Zlatarsky \& Peters (2007) han observado, en corales actuales, como determinadas algas filamentosas se instalan en la parte lesionada provocando un tumor que induce al coral a crear una protuberancia, más o menos cilíndrica, que sobresale de la superficie normal de su esqueleto. Nosotros hemos encontrado protuberancias de este tipo en algunas colonias de Cyathoseris castroi (Fig. 6e) (Alvarez Pérez, 1993).

\section{Anomalías intrapoblacionales e intraespecíficas}

Tipo de colonia

En algunas especies coloniales las coralitas se distribuyen de forma cerioide, como es el caso de Stylophora contorta (Leymerie, 1846) (Fig. 6f). Cuando el aporte detrítico aumenta, se distribuyen de forma pseudoplocoide, de manera que aparecen pequeños canales entre las coralitas que facilitan que la corriente de agua arrastre el sedimento que cae sobre el coral. Este es el caso de la colonia representada en la figura $6 \mathrm{~g}$ (Rodríguez \& GómezHerguedas, 2002).

\section{Forma de la colonia}

Las colonias que viven en zonas de poca profundidad, con escaso aporte detrítico, suelen tener forma más o menos plana, como la colonia de Antiguastraea cvijici de las figuras 6 h e i. Si aumenta la tasa de sedimentación adquieren forma masiva, que soporta mejor las nuevas condiciones ambientales, como la colonia de Antiguastraea cvijici de la figura 6j. Finalmente, si el aporte detrítico sigue aumentando, crecen en forma de columnas redondeadas, que es la forma que mejor soporta elevadas tasas de sedimentación, como la colonia de Actinacis cognata Oppenheim, 1901 de la figura 6k (Pfister, 1985; Kolodziej et al., 2007).

\section{DISCUSIÓN}

El estudio realizado nos muestra cómo ciertos caracteres que presentan los corales pueden variar más o menos ostensiblemente entre los individuos de una misma especie.

Teniendo en cuenta que el único medio de que disponemos para la identificación y clasificación de los corales fósiles es el análisis y estudio de sus esqueletos, cualquier anomalía que puedan presentar es importante para su correcta determinación específica y genérica (Zlatarski \& Martínez-Estalella, 1982; Best et al., 2003; Zlatarski, 2010).

Las anomalías aquí presentadas forman parte de la variabilidad intraespecífica y, por tanto, deberían tenerse en cuenta a la hora de definir una nueva especie. De esta forma evitaríamos la proliferación de nuevas especies y también la multitud de sinonimias que existen actualmente en la sistemática de los corales.

Figura 5. Anomalías en la forma de los corales coloniales. Astrocoenia lobatorotundata. a: (GA 10279c) Castellolí; colonia con los cálices iguales. Astreopora tecta. b: (GA 9718) Castellolí; colonia con los cálices de distinto tamaño. Antiguastraea cvijici. c: (GA 13324) Castellolí; colonia con los cálices de distinta forma y tamaño. Astrocoenia lobatorotundata. d: (GA 12657) Castellolí; colonia con un cáliz gigante. Astreopora tecta. e: (GA 9718) Castellolí; colonia con los cálices regularmente dispuestos; f: (GA 9713) Castellolí; colonia con los cálices dispuestos irregularmente. Antiguastraea cvijici. g: (GA 11562) Castellolí; h: (GA 19616) Castellolí; colonias de la misma especie con los cálices de distinto tamaño. Cyathoseris castroi. j: (GA 8892) Castellolí; colonia con las series calicinales poco marcadas; k: (GA 8654) Pobla de Claramunt; colonia con las series calicinales muy marcadas.

Anomaly in the form of colonial corals. Astrocoenia lobatorotundata. a: (GA 10279c) Castellolí; colony with calices of the like sizes. Astreopora tecta. b: (GA 9718) Castellolí; colony with calices of the differing sizes. Antiguastraea cvijici. c: (GA 13324) Castellolí; colony with calices of the differing forms and sizes. Astrocoenia lobatorotundata. d: (GA 12657a) Castellolí; colony with a giant corallite. Astreopora tecta. e: (GA 9718) Castellolí; colony with regularly arranged calices; $f:$ (GA 9713) Castellolí; colony with irregularly arranged calices. Antiguastraea cvijici. g: (GA 11562) Castellolí; h: (GA 19616) Castellolí; colonies of the like species with calices of differing sizes. Cyathoseris castroi. j: (GA 8892) Castellolí; colony with poorly marked calicinal series; k: (GA 8654) Pobla de Claramunt; colony with clearly marked calicinal series. 

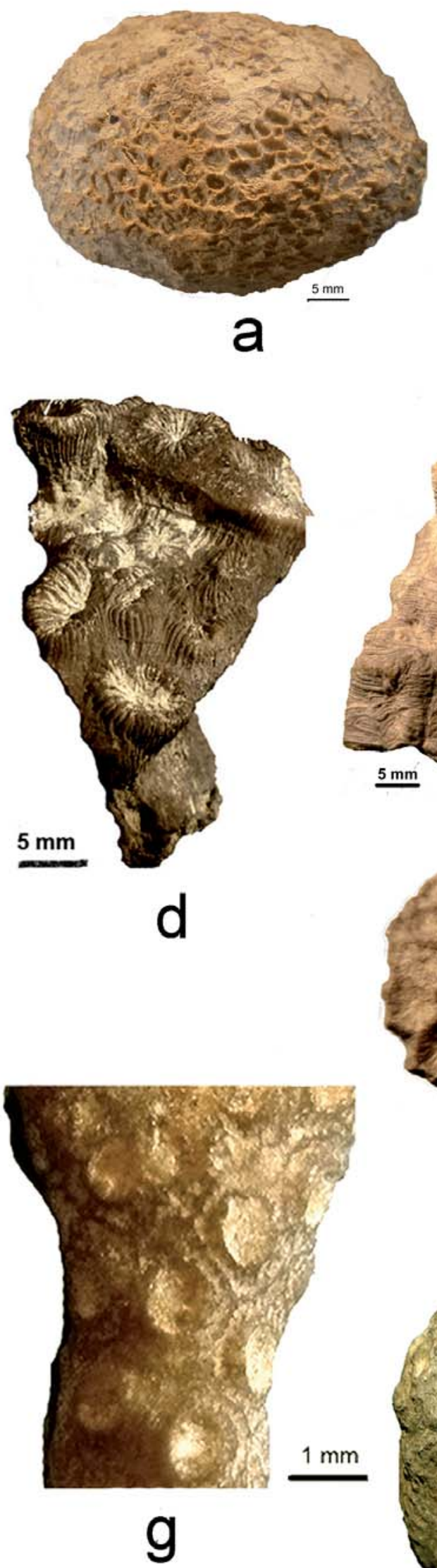

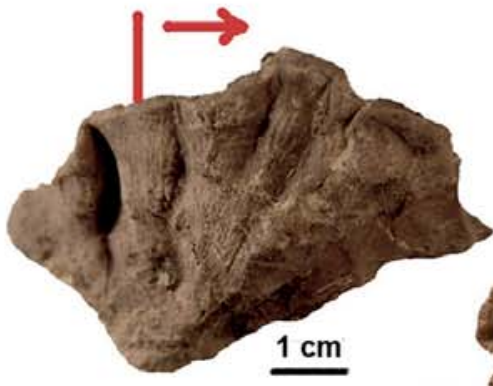

b
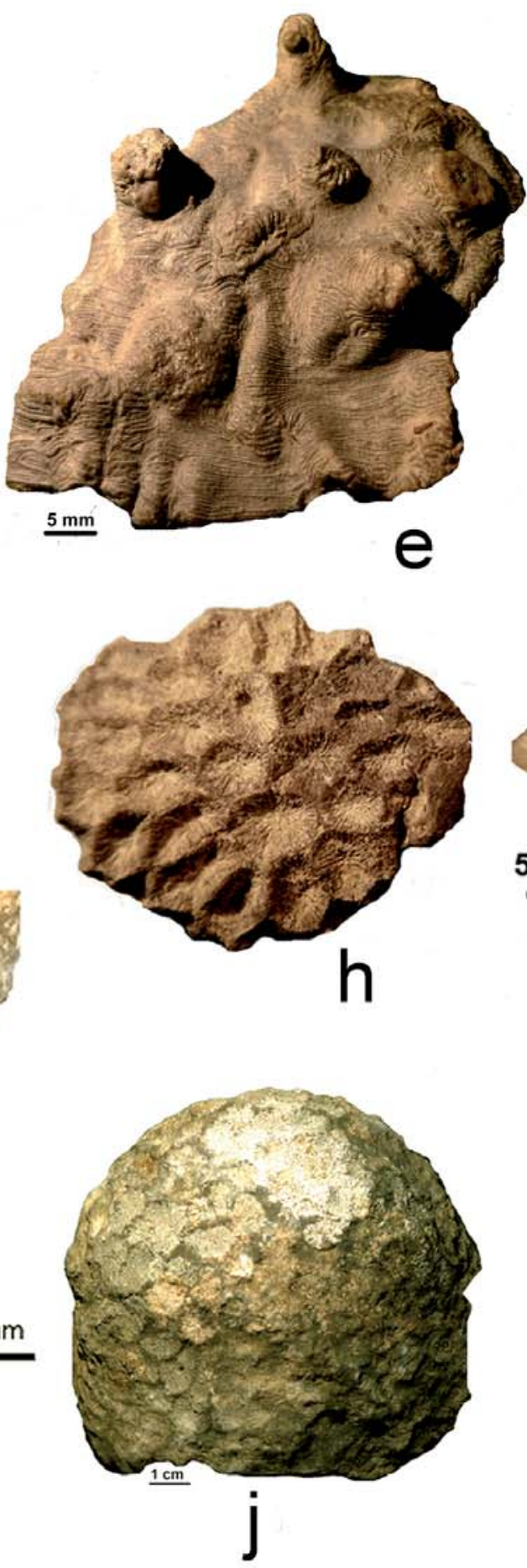

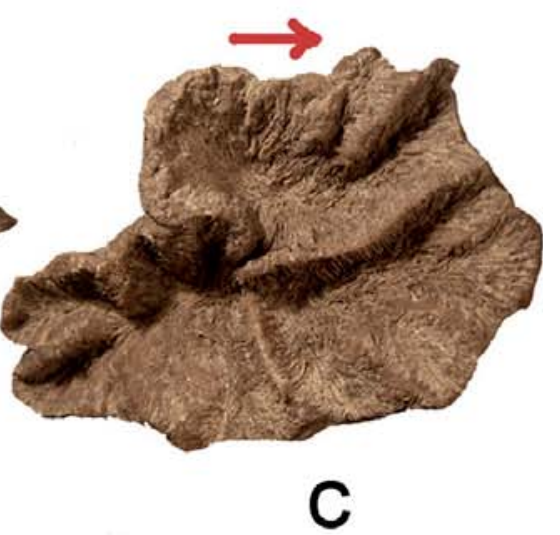

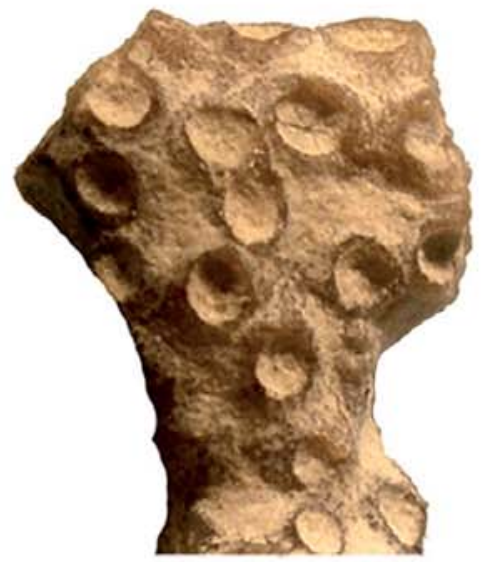

f $2 \mathrm{~mm}$
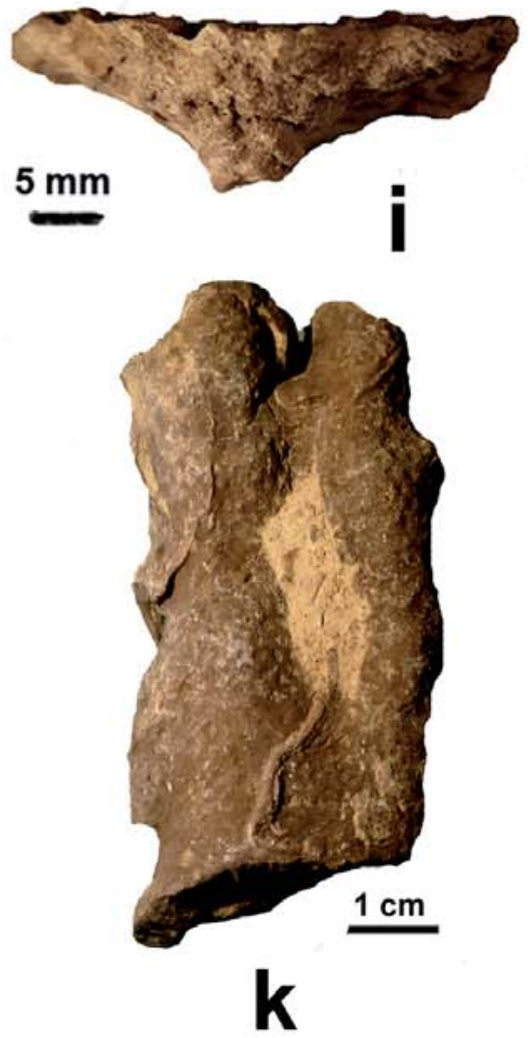


\section{CONCLUSIONES}

El análisis de determinados ejemplares de algunas de las especies coralinas hallados en los afloramientos del Eoceno de Igualada nos ha permitido estudiar una serie de formas anómalas. Estas anomalías se dan en especies solitarias y en especies coloniales. En las especies solitarias se reflejan en la forma del cáliz y en la forma del individuo. En las especies coloniales se reflejan en la forma, tamaño y distribución de las coralitas y en la forma que adquiere la colonia en su proceso de crecimiento y desarrollo. Las causas de dichas anomalías no son del todo conocidas y algunas de ellas no han podido todavía ser demostradas (Zlatarski \& Martínez-Estalella, 1982).

\section{AGRADECIMIENTOS}

Trabajo parcialmente financiado por el Proyecto CGL2005-00404/BTE del Ministerio Español de Educación y Ciencia. Agradecemos las correcciones de los revisores, especialmente las acertadas observaciones del Dr. Sergio Rodríguez, experto en estos temas.

\section{REFERENCIAS}

Alvarez-Pérez, G. 1993. Cnidaria fòssils de la conca d'Igualada. Tesis Doctoral. Universitat de Barcelona, 270 pp.

Alvarez-Pérez, G., Bosence, D., Busquets, P., Darrell, J.G., Franquès, J., Gili, E., Pisera, A., Reguant, S., Rosen, B.R., Salas, R., Serra-Kiel, J., Skelton, P.W., Taberner, C., Travé, A. \& Valdeperas, F.X. 1995. Bioconstructions of the Eocene South Pyrenean Foreland Basin (Vic and Igualada areas) and of the Upper Cretaceous South Central Pyrenees (Tremp Area). VII International Symposium on Fossil Cnidaria and Porifera, Field trip C, 1-68.

Best, M.B., Boekschoten, G.J. \& Oosterbaan, A. 2003. Species concept and ecomorph variation in living and fossil Scleractinia. Palaeontographica Americana, 54, 70-79.
Cascella, A. \& Dinarés-Turell, J. 2009. Integrated calcareous nannofossil biostratigraphy and magnetostratigraphy from the uppermost marine eocene deposists of the southeastern Pyrenean foreland basin: evidences for marine Priabonian deposition. Geological Acta, 17, 281-296.

Costa, E., Garcés, M., López-Blanco, M., Beamundt, E., Gómez-Paccard, M. \& Larrasoaña, J.C. 2009. Closing and continentalization of the South Pyrenean foreland basin (NE Spain): magnetochronological constraints. Basin Research, 452, 1-14.

Fabricius, F. 1964. Aktive Lage-und Ostdverändrung bei der Koloniekoralle Manicina aerolata und ihre fossil Scleractinia. Palaeontographica Americana, 45, 299-324.

Gameil, M. 2005. Palaeoecological implications of Upper Cretaceous Solitary Corals, United Arab Emirates/Oman Borders. Revue de Paléobiologie, Geneve, 24, 515-532.

Jayewardene, D., Donahue, M.J. \& Birkeland, C. 2009. Effects of frequent fish predation on corals in Hawaii. Coral Reefs, 28, 499-506.

Kolodziej, B., Idakieva, V., Ivanov, M. \& Zlatarski, V. 2007. Coral growth forms and growth-interruptions in turbidwater Bioconstructions: examples from lower Cretaceous, Bulgaria. X International Congress on fossil Cnidaria and Porifera, Abstracts, 54-55.

López-Blanco, M. 2006. Stratigraphic and tectonosedimentary development of the Eocene Sant Llorenç del Munt and Montserrat fan-delta complexes (Southeast Ebro basin margin, Northeast Spain). Contributions to Science, 3, 125-148.

Pfister, T. 1985. Coral Fauna and Facies of the Oligocene Fringing reef near Cairo Montenotte (Liguria, Northern Italy). Facies, 13, 175-226.

Rodríguez, S. \& Gómez-Herguedas, A. 2002. Connotaciones paleoecológicas de la morfología externa de los corales rugosos. XVIII Jornadas de la Sociedad Española de Paleontología. II Congreso ibérico de Paleontología, 132-133.

Rosen, B.R., Aillud, G.S., Bosellini, F.R., Clark, N.J., Insalco, E.,Valldeperas, F.X. \& Wilson, M.E.J. 2002. Platy coral assemblages: 200 million years of functional stability in response to the limiting effects of light and turbidity. Proceedings 9th International Coral Reef Symposium, 1, 255-264.

Figura 6. Anomalías en el crecimiento y desarrollo de los corales coloniales. Stylocoenia macrostyla. a: (GA 12654) Castellolí; rotación pasiva. Colpophyllia stellata. (GA 13322) Castellolí; b, c: desplazamiento lateral. Ellipsocoenia bauzai. d: (GA 13311) Castellolí; desplazamiento vertical. Cyathoseris castroi. e: (GA 8611) Puig Aguilera; tumores en forma de verrugas. Stylophora contorta. f: (GA 2806) Castellolí; colonia cerioide. g: (GA 2806b) colonia pseudoplocoide. Antiguastraea cvijici. (GA 3094) Castellolí; h, i: colonia plana; j: (GA 8506) Castellolí; colonia masiva. Actinacis cognate. k: (GA 9441) Puig Aguilera; columnas romas.

Anomaly in the growth and development of colonial coral. Stylocoenia macrostyla. a: (GA 12654) Castellolí; passive rotation. Colpophyllia stellata. (GA 13322) Castellolí; b, c: lateral displacement. Ellipsocoenia bauzai. d: (GA 13311) Castellolí; vertical displacement. Cyathoseris castroi. e: (GA 8611) Puig Aguilera; tumours in wart-like forms. Stylophora contorta. f: (GA 2806) Castellolí; cerioid colony; g: (GA 2806b) pseudoplocoid colony. Antiguastraea cvijici. (GA 3094) Castellolí; h, i: planar colony; j: (GA 8506) Castellolí; massive colony. Actinacis cognata. k: (GA 9441) Puig Aguilera; rounded columns. 
Serra-Kiel, J., Travé, A., Mató, E., Saula, E., FernándezCanadell, C., Busquets, P., Tosquella, J. \& Vergés, J. 2003a. Marine and Transitional Middle/Upper Eocene Units of the Southeastern Pyrenean Foreland Basin (NE Spain). Geologica Acta, 1, 177-200.

Serra-Kiel, J., Mató, E., Saula, E., Travé, A., Fernández-Canadell, C., Alvarez-Pérez, G., Busquets, P., Samsó, J.M., Tosquella, J., Franqués, F., Romero, J. \& Barnolas, A. 2003b. An inventory of the Marine and Transitional Middle/Upper Eocene Deposits of the Southeastern Pyrenean Forelan Basin (NE Spain). Geologica Acta, 1, 201-222.

Sorauf, J.E. 2010. Colonial form, free-living corals, and macroborers from the Pleistocene of South Florida. Palaeoworld, 19, 426-434.
Zlatarski, V.N. 2007. Scleractinian variability and taxonomy: lessons from the past and future perspectives. $X$ International Congress on fossil Cnidaria and Porifera, Abstracts, 106-107.

Zlatarski, V.N. 2010. Paleobiological perspectives on variability and taxonomy of scleractinian corals. Palaeoworld, 19, 333-339.

Zlatarski,V.N. \& Martínez-Estalella, N.1982. Les scléractiniaires de Cuba avec des donnnées sur les organismes associés. Académie bulgare des Sciences, Sofia, 1-472.

Zlatarski, V.N. \& Peters, E.C. 2007. Have you looked for tumors among your fossil material? X International Congress on fossil Cnidaria and Porifera, Abstracts, 107.

Manuscrito recibido: 25 de agosto, 2011 Manuscrito aceptado: 9 de enero, 2012 